\title{
L'appareil génital mâle du Chameau
}

\author{
par M.-A.-F. TAYEB, M.V.Sc.
}

\author{
Traduction : P.-C. BLIN
}

\section{MATÉRIEL D'ÉTUDE ET MÉTHODE}

Le matériel d'étude a été prélevé aux abattoirs du Caire et de Giza. L'appareil génital de 50 animaux fut conservé dans une solution formolée à $10 \%$. La situation des divers organes fut déterminée sur les carcasses, à l'abattoir.

Pour compléter cette étude, deux chameaux furent injectés par voie carotidienne avec la masse d'injection suivante :

arsenic blanc ....................... $200 \mathrm{~g}$

carbonate de sodium .............. $200 \mathrm{~g}$

acide phénique ............... 2,5 kg

glycérine................... 2,5 -

formol à $40 \% \ldots \ldots \ldots \ldots \ldots \ldots \ldots \ldots, 2,5$ -

eau......................... 251

substance colorante .............. Q. S.

2 kilos de sulfate de chaux furent ajoutés à la masse, peu avant l'injection et remués sans cesse au cours de la même opération.

\section{LES ORGANES GÉNITAUX DU MALE}

Les principaux organes génitaux du chameau sont: les deux testicules, les canaux déférents et le pénis.

Les organes génitaux accessoires sont : la prostate et les glandes bulbo-urétrales (de Cowper).

Il est intéressant de noter l'absence des deux vésicules séminales et de l'utricule prostatique (ce dernier est fréquemment rencontré chez le cheval; sa présence est discutée chez le taureau).

\section{Le scrotum}

Aucune description du scrotum du chameau n'a été faite jusqu'ici. Le scrotum occupe une position évidente en région périnéale; il est couvert parliellement par la queue; cependant, on peut l'apercevoir de derrière quand l'animal est en station debout. Le scrotum est de forme ovoïde, de faibles dimensions en valeur absolue et, en valeur relative, à la taille de l'animal. Le tégument scrotal se présente d'ordinaire plus foncé que la peau environnante, rêche, parsemé de poils courts et fins disséminés.
En raison du contact étroit du scrotum et de la région périnéale, il est impossible de décrire dans le sac scrotal un col bien défini. Le raphé scrotal qui constitue la couture médiane du sac scrotal n'est pas bien marqué.

Le scrotum du chameau présente les mêmes couches que celui du cheval (chez le premier ces couches peuvent être facilement différenciées à la dissection).

\section{Le crémaster}

Le muscle crémaster externe est étroit, allongé à son origine sur le fascia iliaca, près de l'attache supérieure du muscle couturier (sartorius); ses fibres divergent progressivement vers le testicule correspondant; il occupe le côté postéro-externe du cordon testiculaire.

\section{Les testicules}

Lesbre (1906) situe le testicule du chameau en région périnéale, à courte distance de l'anus; sous ce rapport, il rappelle le testicule du chien, du chat et du verrat. Lesbre mentionne également la direction antéro-inférieure du grand axe du testicule.

Leese (1927) ajoute que le cordon testiculaire du chameau est plutôt développé et que l'anneau inguinal inférieur est étroit, ne permettant l'introduction que d'un seul doigt.

\section{Travaux personnels}

Les testicules du chameau sont de forme ovoide; la longueur de leur grand axe et leur poids varient chez l'adulte âgé de plus de 3 ans. La longueur du testicule varie de 2,8 à 4 inches $(7 \mathrm{~cm}$ à $10 \mathrm{~cm}$ ); le poids de 80 à 110 grammes. Chaque testicule est placé dans son compartiment scrotal, obliquement de haut en bas et d'avant en arrière. Le bord antérieur est presque droit; l'épididyme lui est rattaché. Le bord postérieur est convexe et libre.

Les extrémités supérieure et inférieure sont arrondies.

A l'extrémité inférieure du testicule, en dessous et en avant de la tête de l'épididyme se trouve l'appendice testiculaire (appendix testis). 
La face exlerne du testicule est convexe et lá face interne est presque plane.

L'épididyme. - Il longe le bord antérieur du testicule, s'étendant de l'extrémité inférieure vers
La queue de l'épididyme est également solidement fixée par le ligament épididymaire à l'extrémité supérieure du testicule.

Le méso testiculaire (mediastinum testis). - Il

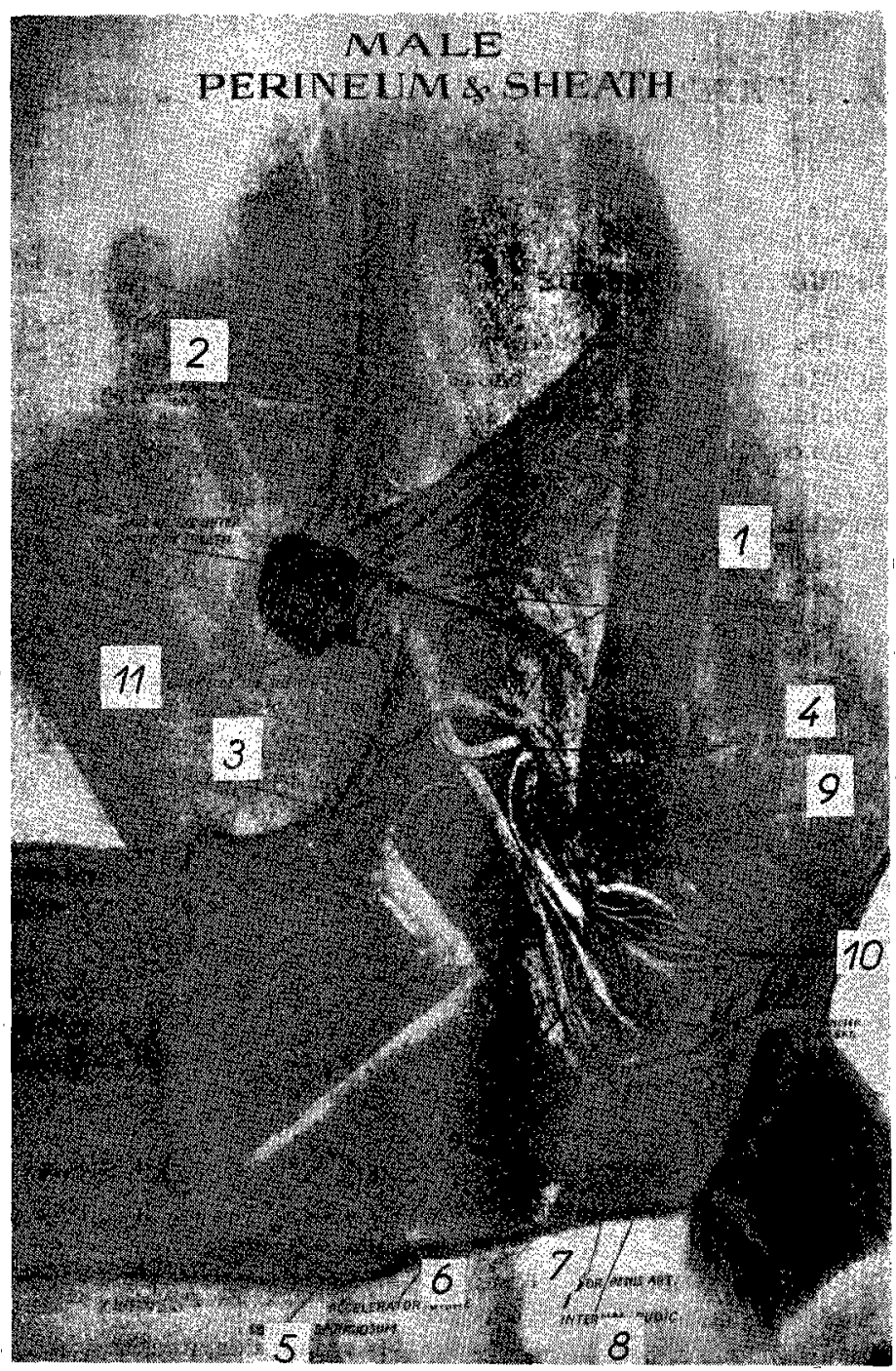

Fig. 1. - Le périné et le fourreau

1. Muscles lateraux du fourreau.

2. Muscles antérieurs du fourreau.

7. Muscle 'rétracteur' du pénis.

3. Testicules ramenés en avant.

8. Artère honteuse interne.

4. Inflexion sigmoïde.

9. Corps caverneux:

5. Corps spongieux.

10. Muscle ischio-caverneux

6. Muscle bulbo-caverneux.

un point situé légèrement au-dessus du niveau de l'extrémité supérieure.

La tête de l'épididyme est solidement rattachée au testicule, là précisément où les cônes efférents du testicule pénètrent dans la tête de l'épididyme. est bien défini et occupe le tiers moyen de la partie centrale de chaque testicule.

Le cordon spermatique. - Il est relativement long (18 à 20 inches environ, soit 45 a $50 \mathrm{~cm}$ ).

A l'extrémité inférieure du testicule, là où il 
débute, il est volumineux, par suite de l'importance du plexus veineux pampiniforme en cet endroit.

L'annean inguinal inférieur. - Il est étroit et ne permet l'introduction que d'un seul doigt; ceci confirme les donnees de Leese.

\section{Les canaux déférents}

Un caractere remarquable des canaux déférents du chameau est leur disposition flexueuse, sauf vers la partie terminale, de leur trajet où ils sont inclus dans le méso interdéférente. chez ie chameau pour les ralsons : suivantes :

1. l'etroitesse absolue des deux anneaux inguinaux inferieur et supérieur;

2. l'ampleur et la position des ganglions inguinaux superficiels en regard de l'anneau inguinal inférieur ;

3. la compression de la portion inguinale du cordon spermatique par les ganglions précités dans le territoire de l'anneau inguinal inférieur.

Il en résulte que, si l'une quelconque de ces hernies se produit, sa réduction est difficile (pour les raisons mentionnees et aussi par suite de l'existence

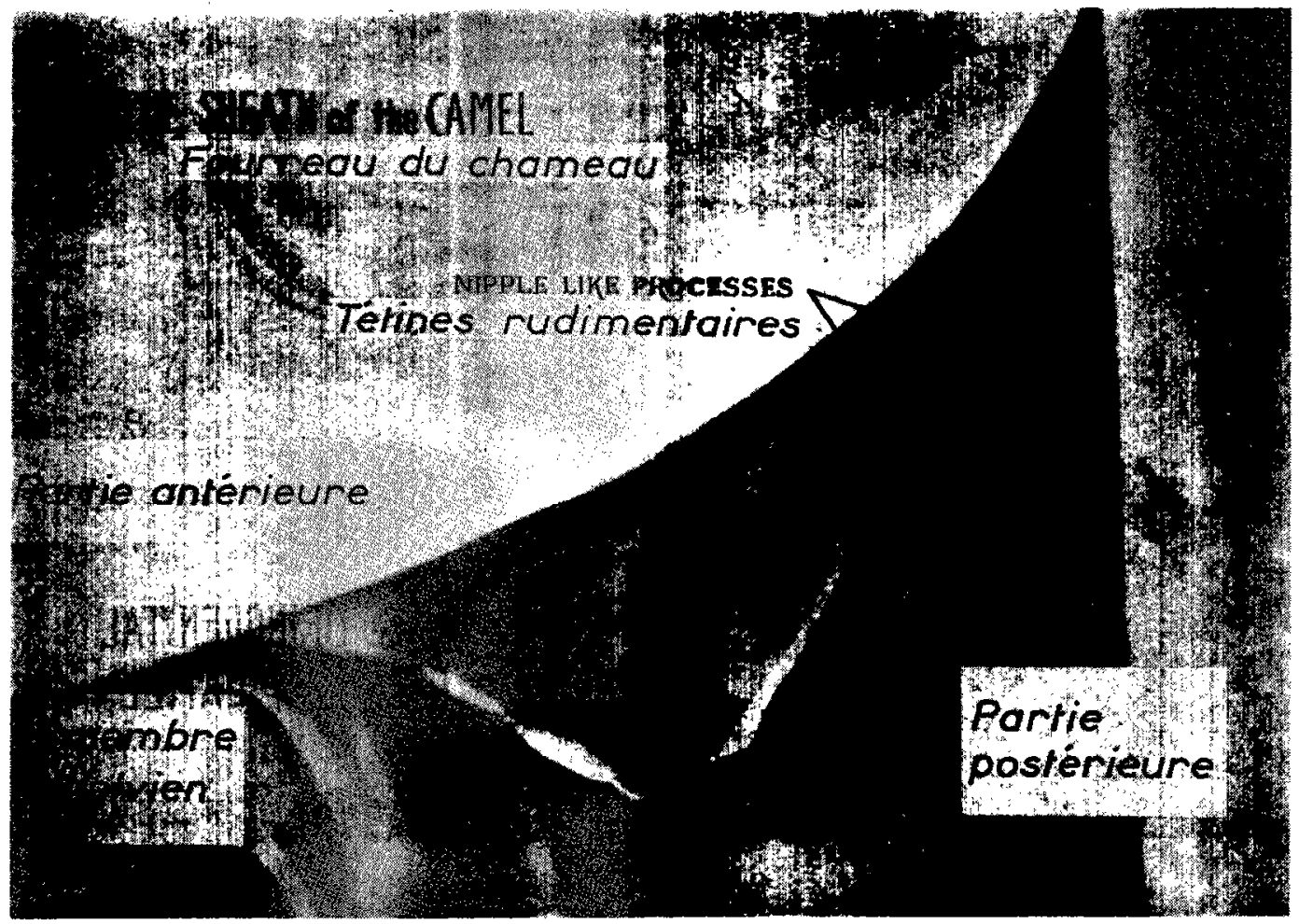

Fig. 2. - Organes genitaux

Fourreau (ou prépuce) présentant une sorte de rétrécissement à son orifice (une des formes varjèes de rétrécissement)

Les ganglions inguinaux superficiels. - Leese (1927) a noté la présence de ganglions inguinaux superficiels en avant du muscle droit interne (gracilis). Chez tous les sujets disséqués, les ganglions inguinaux superficiels (au nombre de 2) recouvraient partiellement l'anneau inguinal inferieur et le cordon spermatique à ce niveau.

Ces ganglions sont volumineux et mesurent 12 à 13 centimètres de longueur, 4 à 5 centimètres de largeur et 0.5 à 2 centimètres d'épaisseur.

En conclusion, on peut dire que les hernies, qu'elles soient scrotales ou inguinales, sont rares d'une importante couche de muscles, préputiaux.

\section{Le pénis et le fourrean}

Il convient de faire la description du fourreau du chameau avant celle du pénis.

a) Le fourreau. - Lesbre (1906) décrit le fourreau du chameau comme une volumineuse saillie conique, charnue, pendant de l'abdomen, telle une glande mammaire énorme. Il est perforé d'un orifice étroit, ne laissant passer que le bout du doigt, situé à son extrémité libre inférieure, dirigé vers l'arrière. La touffe de poils qu'on rencontre à l'orifice 
préputial du taureau n'existe pas chez le chameau.

Lesbre note encore la présence de muscles rétracteurs et protracteurs du fourreau.

\section{Travaux personnels}

L'examen du fourreau montre sa ressemblance structurale et non morphologique avec celui des autres animaux domestiques.
Le tégument préputial est de couleur plus foncée que la peau environnante et présente de. courts poils fins et disséminés. A la partie supérieure du fourreau et, assez souvent, immédiatement au-dessus de l'orifice préputial, on rencontre une paire de papilles de chaque côté. Ces papilles sont généralement considérées comme des tétines rudimentaires.

Des muscles agissant sur le fourreau, lui sont

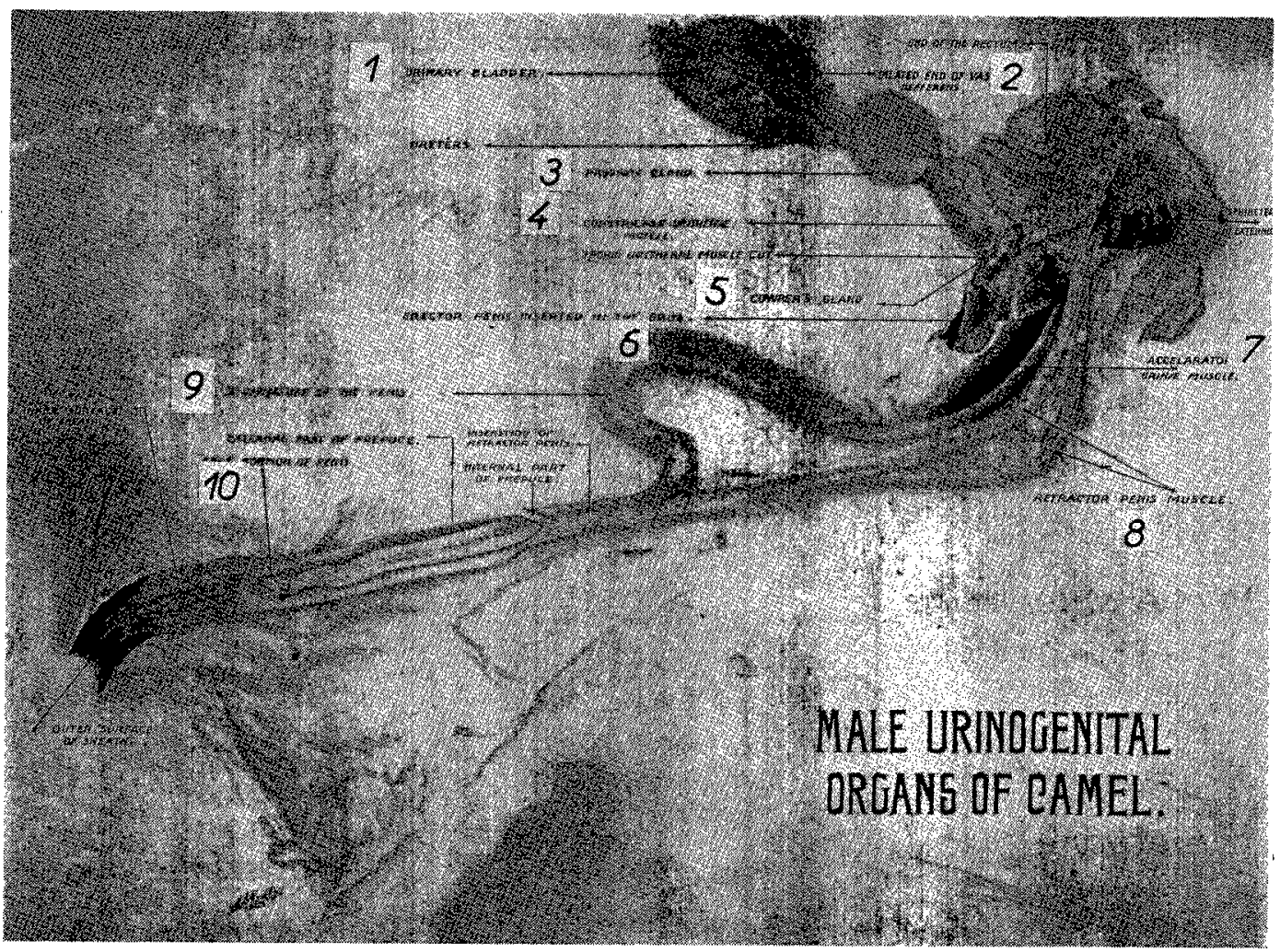

Fig. 3.
I. Vessie.
2. Renflement terminal du canal déférent.
3. Prostate.
4. Muscle constricteur de l'urètre.
5. Glande bulbo-urétrale (de Cowper).

Il est formé d'une couche externe ou pariétale et d'une couche interne ou viscérale réfléchie sur le pénis, à son extrémité libre.

En dehors de la phase d'érection, le pénis du chameau est caché dans le fourreau qui forme un revêtement triangulaire, vaste, massif, suspendu, quand il est vu par côté. L'extrémité inférieure, libre, conique, présente l'orifice préputial qui n'admet que le passage de l'index.

L'orifice préputial étant dirigé en arrière, le jet d'urine se fait dans la même direction, lors de la miction.
6. Muscle ischioücaverneux.
7. Muscle bulbo-caverneux (accélérateur).
8. Muscle rétracteur du pénis.
9. Inflexion sigmoide.
10. Partie libre du pénis.

annexés. Ils modifient sa forme et sa position. Dans l'ensemble, ces muscles apparaissent sous forme de bandes musculaires aplaties incorporées au fascia superficiel (fascia superficialis) en région souscutanée. Ces bandes musculaires prennent leur origine au voisinage du fourreau, mais toutes convergent vers l'orifice préputial au niveau duquel elles s'inserent.

Les muscles du fourreau forment 3 groupes :

1. Les muscles antérieurs du fourreau (protracteurs de Lesbre) représentent deux bandes musculaires, bien délimitées, qui, à partir de 
l'ombilic, s'étendent en arrière, parallèlement l'une par rapport à l'autre, de chaque côté de la ligne ventrale médiane de la tunique abdominale, pour se terminer au niveau de l'orifice préputial.

2. Les muscles postérieurs du fourreau (rétracteurs de Lesbre) sont plus courts que les précédents et : $s$ 'étendent d'un point situé juste en avant du scrotum à l'orifice préputial.

3. Les muscles latëraux du fourreau, de chaque côté, comprennent 3-4 bandes musculaires étroites de longueur variable convergeant en définitive vers l'orifice preputial

La plus longue de ces bandes musculaires prend origine en région inguinale, au-dessous des ganglions inguinaux superficiels.

Les fibres de tous les muscles décrits interfèrent pour constituer une importante masse conique à l'entour de l'orifice préputial.

C'est par l'action de ces muscles que le fourreau peut être porté, soit en avant, soit en arriere, lors de l'érection ou de la miction, respectivement.

Les muscles du fourreau exercent encore une dilatation ou une constriction de l'orifice préputial.

b) Le pénis. - Lesbre (1906) montre la ressemblance du pénis du chameau avec celui du taureau et note l'aspect crochu du gland. Il ajoute que l'inflexion sigmoïde du pénis se situe en avant des testicules et que le muscle bulbo-caverneux (accelerator urinæ) s'étend jusqu'à cette inflexion.

Leese (1927) est en accord avec Lesbre, quant à la ressemblance d'ensemble du penis du chameau avec celui du taureau. Il signale que la seule différence importante tient à la forme du gland qui offre l'aspect d'un crochet dont la convexité est superieure et légèrement inclinée par côte. La base du gland est formée par une expansion cartilagineuse incurvée.

\section{Travaux personnels}

L'examen du pénis confirme les donnèes des auteurs précédents. La ressemblance entre le pénis du taureau et celui du chameau ne se borne qu'à leur forme et à leur structure. La différence essentielle, toutefois, réside dans le fait que le gland est pointu chez le bœuf, crochu chez le chameau.

Par ailleurs, l'inflexion sigmoïde occupe une situation différente en rapport avec la situation différente des testicules chez ces deux animaux. Chez le chameau, l'inflexion sigmoide est préscrotale; chez le bœuf elle est postscrotale.

Le pénis du châmeau est légèrement comprimé d'un côté à l'autre dans sa portion postsigmoide, arrondi dans sa portion présigmoïde.

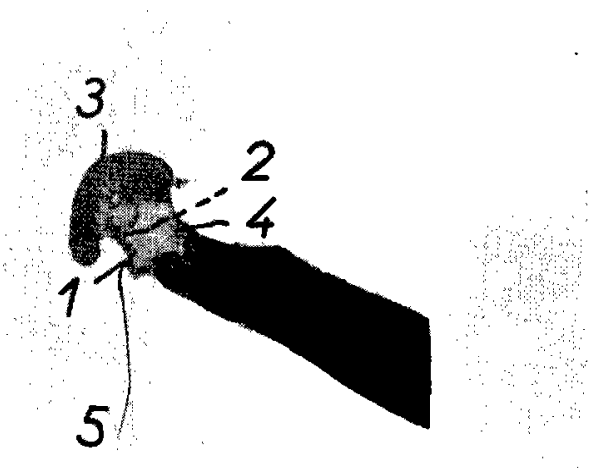

Fig. 4. - Extrémité libre antérieure du pénis

1. Orifice externe de l'urètre.

2. Papille conique.

3. Gland recourbé en crochet.

4. Col du gland.

5. Un fil introduit dans l'urètre montre le très faible calibre de celvi-ci à sa terminaison.

Son épaisseur décroît de son origine sur l'arcade ischiatique, en arrière, à son extrémité libre en avant. La longueur moyenne du pénis, chez un animal adulte est de 17,5 centimètres ( 7 inches) dans sa portion post-sigmoilde, 25 centimètres ( 10 inches) au niveau de l'inflexion sigmoide, 17,5 centimètres (7 inches) dans sa portion présigmoïde.

Au niveau de la racine du pénis, le diametre de l'organe est de 2,5 centimetres ( 1 inche) environ et juste en arrère du col du gland, il n'est que de 1 centimetre.

Le pénis du chameau est formé de 2 racines qui prennent leur origine au niveau de l'arcade ischiatıque près de la ligne médiane et réunies l'une à l'autre apres un court trajet pour constituer la majeure partie du corps du pénis.

Craque racine est enfouie dans le muscle ischiocaverneux. Ce dernier est comprimé latéralement et se présente moins développé que celui du cheval: il prend origine sur l'arcade ischiatique, en commun avec la racine du pénis, sur la tubérosité ischiatique (luber ischii) et sur la face adjacente du ligament sacro-sciatique; il s'effile progressivement à son insertion sur la racine du pénis et la partie adjacente du corps du pénis.

Le corps caverneux du pénis (corpus cavernosus penis) est fait d'un tissu érectile qui est moins important que celui du cheval et celui du taureau.

Le tissu érectile du pénis, l'urètre et le corps caverneux de l'uretre (corps spongieux) associés à lui sont tous enfermes dans une epaisse couche fibreuse, la tunique albuginee (tunica albuginea). 


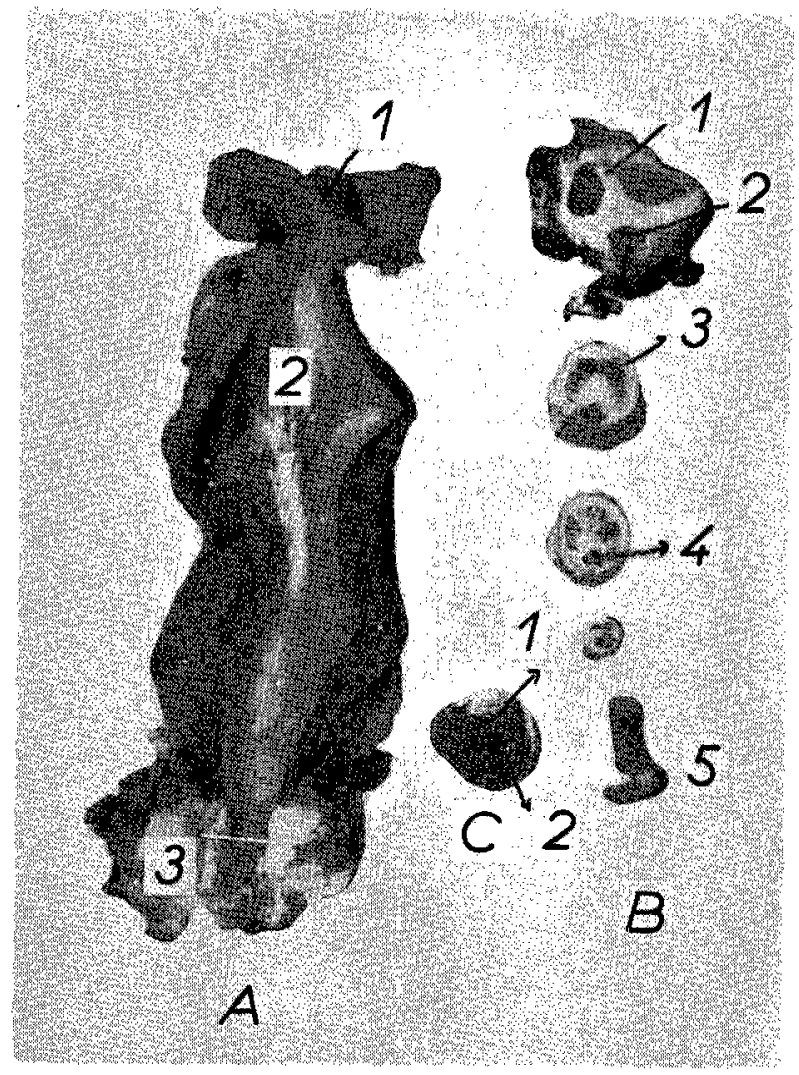

Fig. 5. - Orgànes génilaux Vue interne de l'urètre pelvien (plafond)

A. - I'. Portion de vessie.

2. Veru montanum présentant les orifices des canaux éjaculateurs

3. Partie terminale rétrécie de l'urètre pelvien.

B. - Coupes segmentales du pénis.

1. Septum central.

2. Portion cxtrapelvienne de l'urètre.

3. Coupe plus antérieure.

4. Coupe plus antérieure.

5. Gland.

c. - Coupe segmentale de l'urètre pelvien montrant sa lumière et mettant en évidence, du côté ventral, I'épaisseur du muscle urétral.

De la face interne de la tunique partent des septa qui délimitent de multiples espaces caverneux qui sont plus petits que ceux rencontrés chez le cheval ou le taureau. Ces septa se réunissent sur la ligne médiane pour former un septum penis mal défini; ce dernier n'est rencontré que dans la portion postérieure de l'organe.

Par suite de l'épaisseur et de la naturc fibreuse de la tunique albuginée, des dimensions réduites des espaces caverneux, le pénis du chameau ne peut que se distendre faiblement au cours de l'érection, le fait essentiel étant l'accroissement de la rigidité de l'organe sur une coupe segmentale, le corps caverneux de l'urètre (corpus cavernosus urethræ) est très petit.
Il cumprend une très falble quantite de tissu érectile renfermant en son milieu l'urètre lui-même. Le corps caverneux de l'urètre est entouré par-dessus et par côté du corps caverneux du pénis (corpus cavernosus penis).

Tie gland est séparé du corps par un col bien marqué. Il n'y a rien à ajouter aux données des auteurs précités. A l'examen ınicroscopique, le gland présente de vastes espaces caverneux, des cellules cartilagineuses, du tissu fibro-élastique.

Ainsi le gland, par sa structure même, semble capable de subir une certaine élongátion, an cours de l'érection; 'en fait, au cours du coilt le pénis devient rectiligne, quelque peu pointu à l'extrémité.

Les muscles du pénis :

1. L'ischio-caverneux (ischio-cavernosus = erector penis) a été décrit.

2. Le rétracteur du pénis (retractor penis) rappelle celui du taureau.

3. Le bulbo-caverneux (bulbo-cavernosus = accelerator urinæ) représente la portion prolongée du muscle urétral qui entoure la portion pelvienne de l'urètre.

Il. est fait de deux moitiés latérales, séparées l'une de l'autre par un raphé médian issu de leur aponévrose d'enveloppe. Chaque moitié est large à l'origine, offre 2,5 centimètres ( 1 inch) d'épaisseur environ et s'effile progressivement vers l'insertion sur le côté du corps caverneux du i pénis. Contrairement à l'assertion de Lesbre, ce muscle n'atteint pas l'inflexion sigmoïde.

\section{L'URÈTRE}

Lesbre (1906) rapporte la terminaison du canal dé l'urètre, sous le gland, à l'extrémité d'un appendice'entouré de papilles triangulaires. Il indique également que la portion intrapelvienne de l'urètre est enveloppée d'un sphincter épais. Leese (1927) écrit qu'on peut introduire un manche de scalpel dans cette même portion intrapelvienne, tandis qu'on peut tout juste introduire, un cathéter pour chien dans la portion extrapelvienne, progressivement rétrécie vers l'extrémité.

\section{Travaux personnels}

L'urètre du chameau s'étend du col de la vessie au gland où il s'ouvre par l'orifice urétral externe, immédiatement au-dessous de la face inférieure concave du gland.

La portion pelvienne de l'urètre mesure 12,5 à 
14 centimètres ( 5 à 6 inches) de longueur du col de la vessie à l'arcade ischiatique.

Le péritoine ne recouvre que la moitié antérieure de la portion pelvienne de l'urètre. après s'être réfléchi de la paroi rectale pour former le cul-de-sac recto-génital.

A l'origine, le diamètre de l'urètre pelvien est de 2 centimètres environ; la lumière du canal urétral se rétrécit vers la jonction avec la portion extra-pelvienne.

La partie rétrécie de l'urètre reçoit les glandes bulbo-urétrales (de Cowper).

En dedans, l'uretre pelvien présente un verumontanum (colliculus seminalis); celui-ci, sous forme d'une éminence semi-circulaire, occupant le plafond du canal, est situé à 2,5 centimètres ( 1 inch) environ de l'orifice urétral interne et se développe d'avant en arrière sur 1,8 centimètres ( $3 / 4$ inch) environ de longueur. Le bord arrondi de l'éminence regarde vers l'arrière. La portion extrapelvienne de l'urètre se réduit progressivement dans son diamètre. A l'extrémité libre du pénis, elle se termine par l'orifice urétral externe qui ne peut recevoir qu'une très fine sonde. Cet orifice est situe à 2-3 millimètres en arrière de la portion incurvée du pénis, entouré d'une papille conique.

\section{Les muscles de l'urètre :}

1. Le muscle urétral (constrictor urethræ) entoure l'urètre pelvien ventralement et latéralement. Il est très épais dans sa partie ventrale; il s'amincit progressivement sur les côtés, de sorte qu'il a une forme en croissant sur les coupes segmentales.

2. Le muscle ischio-urétral est issu de l'arcade ischiatique et vient s'insérer sur la partie postérieure de l'urètre pelvien, en recouvrant les glandes bulbo-urétrales.

3. Le muscle bulbo-caverneux (bulbo-cavemosus) a été décrit avec les muscles du pénis.

\section{Les organes annexes de l'appareil génital}

Lesbre (1906) mentionne la présence de glandes de Cowper et l'absence de vésicules séminales chez le chameau. Il indique aussi que la prostate du chameau rappelle celle du cheval.

Leese (1927) confirme l'absence des résicules séminales chez le chameau.

La prostate. - La prostate du chameau est constituée par une masse discoïde simple, de couleur jaune foncé (en opposition avec Lesbre. qui la compare à celle du cheval).

Chez le cheval, la prostate comprend deux lobes latéraux réunis par un isthme.

La glande est situee sur le bord supérieur de la

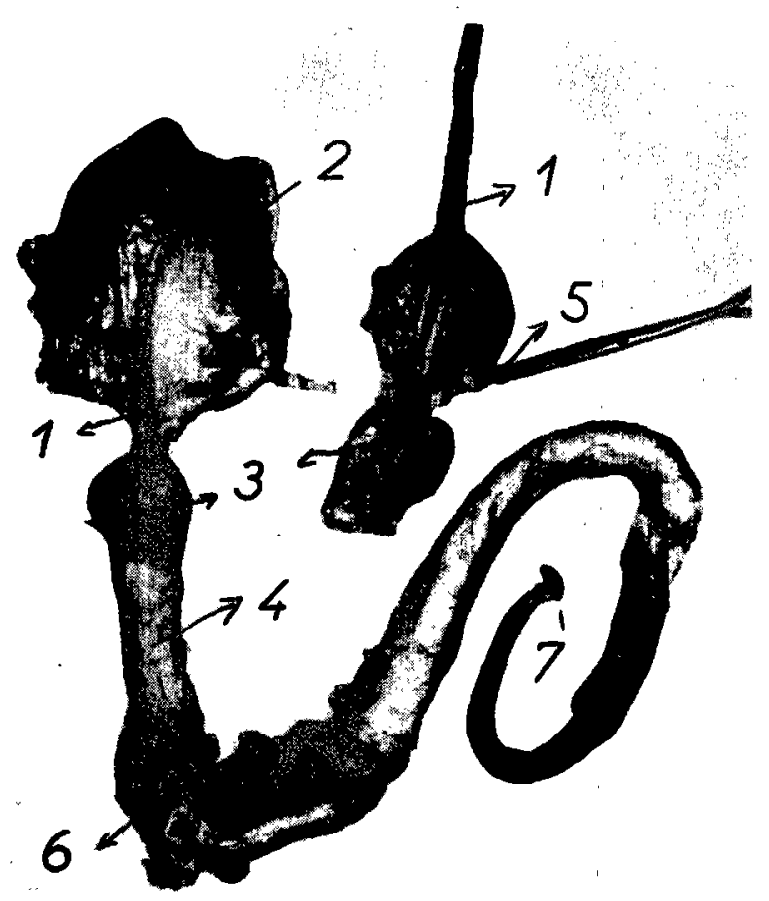

Fig. 6. - Organes génitaux

1. Renflement terminal du canal déférent (noter l'absence de vésicule séminale et d'utricula prostatiqua).

2. Vessie.

3. Prostate.

4. Urètre pelvien.

5. Uretère.

6. Glande de Cowper sectionnée pour montrer son canal unique.

7. Gland.

première portion de l'urètre pelvien, au niveau du col vésical. Elle mesure 3,1 et 5 centimètres $(1,5$ inch et 2 inches) dans ses diamètres longitudinal et transversal, respectivement.

Les glandes bulbo-urétrales (de Cowper). Elles sont au nombre de deux ; chacune est située de chaque côté de l'urètre pelvien à sa terminaison, en regard de l'arcade ischiatique. Elles sont de couleur blanchâtre, en forme d'amandes; le grand axe ( $2.5 \mathrm{~cm}$, soit 1 inch, environ) est dirigé en arrière et en bas; le petit axe mesure 1,2 centimètres, soit $1 / 2$ inch, environ.

Le conduit unique de chaque glande se dirige en arrière et en bas et s'ouvre dans la portion terminale de l'urètre pelvien.

\section{L'irrigation et l'innervation de l'appareil génital mâle du chameau}

L'irrigation. - Les données de Lesbre (1906) 
relatives "à l'irrigation de l'appareil génital du chameau sont peu satisfaisantes.

Il mentionne seulement la division de l'artère

\section{Travaux personnels}

- La distribution de l'artère iliaque interne rappelle celle du cheval.

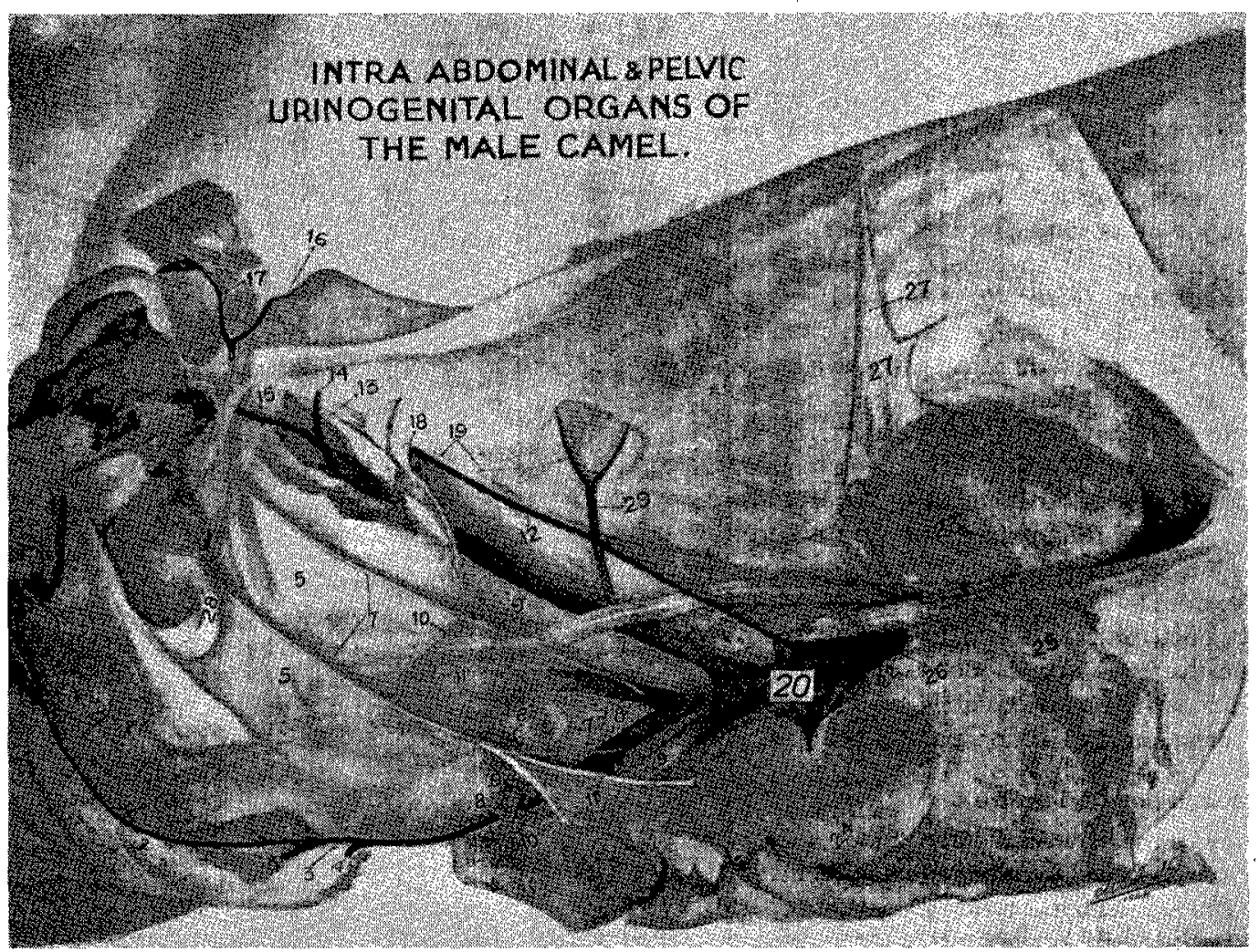

Fig. 7. - Organes génitaux

\author{
1. Urètre pelvien. \\ 2. Artère honteuse externe. \\ 3. Artère ischiatique. \\ 4. Artère fessière antérieure. \\ 5. Ligament latéral de la vessie. \\ 6. Rectum. \\ 7. Artère ombilicale. \\ 8. Artère iliaque interne. \\ 9. Artère iliaque externe. \\ 10. Canal déferent. \\ 11. Uretères. \\ 12. Artère et veines spermatiques. \\ 13. Cordon testiculaire dans le canal inguinal. \\ 14. Artère abdominale postérieure.
}

15, 16. 17. Artère honteuse externe et ses branches. 18. Anneau inguinal interne.

19. Nerfs inguinaux.

20. Aorte superieure.

21. Artère grande mésentérique.

22. Artère et veine rénales.

23. Rein droit.

24. Rein gauche.

25.

26.

27. Enveloppe péritonéale du rein droit.

28. Ligament inférieur de la vessie.

29. Artère circonflexe iliaque. iliaque interne, en regard de la petite échancrure sciatique en deux branches : l'artère honteuse interne et l'artère ischiatique. La première se comporte comme chez les autres animaux domestiques.

Il en est de même, dit-il, pour l'artère testiculaire; quant à l'artère obturatrice, elle est rudimentaire comme celle du boeuf.
- Lartere spermatique interne (testiculaire ou spermatique) :

L'artère irrigue le testicule et l'épididyme; elle prend origine à partir de l'aorte postérieure, en arrière de l'émergence des artères rénales et, à 7,5 à 10 centimètres ( 3 à 4 inches) environ de sa terminaison.

Le trajet de l'artère spermatique interne rappelle 
celui rencontré chez d'autres animaux domestiques. - L'artère spermatique externe (artère du cordon) :

Elle a une origine variable. Chez quelques sujets, l'artère circonflexe iliaque est remplacée par deux vaisseaux qui représentent les branches de terminaison habituelles quand elle est simple. Dans ces cas, l'artère du cordon naît à partir du vaisseau postérieur connu sous le nom d'artère circonflexe iliaque postérieure chez les bovins. Dans d'autres cas, quand l'artère circonflexe est simple, l'artère du cordon naît dans l'angle formé par l'artère iliaque externe et l'artère circonflexe iliaque.

Le vaisseau est très fin ; il assure la vascularisation du cordon spermatique.

- L'artère honteuse externe:

Ce vaisseau naît de l'artère prépubienne (tronc pudendo-épigastrique).

Le tronc est issu de l'artère iliaque externe; mais il ne naît pas en commun avec l'artère fémorale profonde, comme c'est le cas chez le cheval.

Le vaisseau se termine en se divisant en deux branches; l'artère abdominale postérieure et l'artère honteuse externe.

L'artère honteuse externe émerge au niveau de la commissure interne de l'anneau inguinal inférieur où elle émet une branche importante pour le ganglion inguinal superficiel; puis, après un court trajet, elle se divise en deux branches; l'une se dirige en avant pour irriguer le prépuce ; l'autre se dirige en arrière pour irriguer le scrotum.

De cette distribution il résulte que, contrairement à ce qu'on voit chez le cheval, l'artère honteuse externe du chameau ne fournit pas l'artère dorsale du pénis.

- L'artere honteuse interne:

C'est l'artère principale des organes génitaux du chameau.

Elle naît à partir de l'artère iliaque interne, un peu en arrière de la grande échancrure sciatique

Elle donne les branches suivantes :

1. des branches vésicales, au nombre de 2-3, pour la vessie;

2. une branche prostatique;

3. une branche urétrale pour l'urètre pelvien;

4. l'artère du bulbe urétral, atteignant le bulbe du corps caverneux de l'urètre;

5. l'artère profonde du pénis (profonda penis), pour le corps caverneux du pénis.

Après avoir émis ces branches, l'artère honteuse interne poursuit son trajet sur le bord dorsal du pénis, comme artère dorsale du pénis.

Il est intéressant de constater que l'artère profonde du pénis naît de l'artère honteuse interne et non de l'artère honteuse externe, comme c'est le cas chez les équidés.
Une telle distribution rappelle celle 'rencontrée chez les bovins.

Mieux, l'artère dorsale du pénis est le prolongement de l'artère honteuse interne.

L'innervation. -- L'inncrvation du pénis est assurée par le nerf honteux constitué par des rameaux issus des branches primaires inférieures des $3^{\mathrm{e}}$ et $4^{\mathrm{e}}$ nerfs sacrés.

Il atteint le bord dorsal du pénis, accompagné de l'artère honteuse interne:

Les testicules sont innervés par des rameaux issus des plexus rénal et mésentérique postérieur du sympathique.

Le fourreau est innervé par des rameaux du nerf spermatique externe qui accompagne l'artère honteuse externe.

Le nerf spermatique externe vient lui-même de la branche primaire inférieure du $3 \mathbf{e}$ nerf lombaire.

Ce nerf donne un rameau qui se réunit à une branche issue du 2 e nerf lombaire, pour constituer les nerfs inguinaux destinés à innerver le cordon spermatique.

\section{Résumé}

Les organes génitaux du chameau ont été étudiés sur 3 animaux injectés et 50 appareils conservés. Quelques organes ont été examinés. en place aux abattoirs du Caire et de Giza.

1. L'irrigation des organes génitaux mâles du chameau diffère peu de celle du taureau, mais diffère au contraire beaucoup de celle du cheval.

2. L'innervation rappelle celle des autres animaux domestiques.

3. Le testicule présente un méso testiculaire facile à mettre en évidence sur des coupes sagittales.

4. Le cordon spermatique est long, le canal déférent est petit et flexueux tout au cours de son trajet.

5. Le penis a une forme en crochet recourbé; l'examen au microscope róvèlc à son nivcau la présence de cellules cartilagineuses.

6. Vésicules séminales et utérus prostatique sont absents.

7. Le prostate se distingue de celle du cheval et du taureau par son unicité.

8. Le fourreau est pendant, de forme triangulaire; l'orifice est orienté vers l'arrière; des muscles du fourreau surnuméraires inconnus chez les autres animaux domestiqués, existent chez le chameau.

(College of veterinary Medicine - Fouad lar University Le Caire, Egypte). 


\section{BIBLIOGRAPHIE}

1. M.-F.-X. LESBRE. - Recherches anatomiques sur les Camélidés. Extr. from $\Lambda$ rchives du Museum d'Hist. Nat., Lyon, 1906.
2. A.-S. LEESE. - A treatise on the one-humped camel. Published and printed by Haynes and Sons, Maiden Lane, Stamford, Lincolnshirc, 1927. 